\title{
Rapid quantification of Psilocybin with reversed-phase HPLC and single-wavelength detection
}

\author{
Alexzander Samuelsson², Eric Janusson ${ }^{1}$, Sajni Shah ${ }^{1}$, Markus Roggen ${ }^{1 *}$ \\ ${ }^{1}$ DELIC Labs, 3800 Wesbrook Mall, Vancouver BC, Canada V6S 2L9 \\ ${ }^{2}$ Havn Life Sciences, 2200-885 West Georgia Street, Vancouver, British Columbia, Canada \\ V6C 3E8
}

\begin{abstract}
The alkaloid psilocybin (4-phosphoryloxy- $\mathrm{N}, \mathrm{N}$-dimethyltryptamine) and the neurologically active psilocin (4-hydroxy- $\mathrm{N}, \mathrm{N}$-dimethyltryptamine) are the foremost compounds of pharmaceutical interest in Psilocybe mushrooms. As these compounds are infrequently analyzed in analytical labs, validated methods for rapid purity analysis are lacking. Newfound therapeutic use has invigorated academic and commercial interests in the molecules and new methods of production and available products are expanding. As a result, high-throughput methods of analysis for psilocybin must be improved to promptly determine chemical differences between mushroom genera or other sources of psilocybin and psilocin, as well as refined product purity. To address this, we developed an inexpensive HPLC technique for the efficient quantification of psilocybin and psilocin by using readily available equipment and dilute reagents. Aqueous ammonium formate $(0.143 \mathrm{mM})$ was found to be preferable over techniques with much higher buffer concentrations or stronger acids for controlling psilocybin Zwitterion resolution. The chromatographic run time satisfied high-throughput analytical requirements with an efficient total runtime under 2 minutes. A standard octadecyl silica $\left(C_{18}\right)$ column provided excellent resolution between psilocybin and psilocin signals. The quality of the method was validated using certified analytical reference standards and was found to be accurate (3.5\% bias, Psilocybin), reliable $(0.32 \%$ RSD), and efficient (Psilocybin $k^{\prime}=1.78$ ).
\end{abstract}

\section{Keywords}

- Psilocybin, Psilocin

- Method development

- High performance liquid chromatography

- High-throughput experimentation 


\section{Introduction}

Psilocybin and psilocin are indole alkaloids found in a variety of mushroom-forming fungi commonly referred to in North America as magic mushrooms. These alkaloid indoles are naturally occurring agonists of 5-hydroxy tryptamine $2 \mathrm{~A}$ receptors located in the mammalian nervous system. In humans, the compounds cause a variety of psychotropic effects which can include altered states of consciousness and perception in addition to auditory and visual hallucinations with minimal systemic effects (Passie et al., 2002; Daniel and Haberman, 2017).

Psilocybin was first extracted and sold for therapeutic research in 1958 when Albert Hoffman, Sandoz chemist and discoverer of LSD, isolated the active ingredient from Psilocybe Mexicana (Geiger et al., 2018). Soon after, Sandoz sold synthetic psilocybin under the trade name Indocybin Sandoz for use in psychotherapeutic research. Sandoz ended production in 1965 as the use of LSD and psilocybin became increasingly popular as a recreational drug (Carhart-Harris and Goodwin, 2017). Prohibition began in the 1970s when Psilocybin became a schedule 1 compound in 1971 under the UN convention on drugs. Around the same time, Psilocybin was classified as a Schedule I substance under the USA Controlled Substance Act which meant the drug had high potential for abuse, no accepted medical use, and a lack of accepted safety under medical supervision to US regulators. The Schedule I classification made psilocybin illegal to manufacture, distribute, or possess. For these legal reasons, medical research into the clinical uses of psilocybin lost momentum until the 1990s (Vollenweider, 1998; Gouzoulis-Mayfrank et al., 1999). Interest in psilocybin has recently reignited due to its potential therapeutic capabilities in treating psychological disorders (Cormier, 2020). Since it is an established agonist of serotonin receptor 2a, psilocybin is being investigated as a pharmacological agent for the treatment of serotonin-mediated neurological disorders (Andén et al., 1971; Vollenweider, 1998; Gouzoulis-Mayfrank et al., 1999; Carhart-Harris et al., 2021). These include investigations for the treatment of depression, cancer-related anxiety, obsessive-compulsive disorder, alcohol addiction, and nicotine addiction (Geiger et al., 2018; Fricke et al., 2019).

Psilocybin production is now possible on a significant scale. The synthesis of psilocybin first described by Hoffman was recently refined for a larger scale by Shirota et al. (Shirota et al., 2003). New methods of biosynthetic production from tryptophan, for 
example, have been described recently (Shirota et al., 2003; Molecule of the Week Archive - Psilocybin, 2017). Bioengineered production was also determined to be feasible using $E$. coli, S. cerevisiae, and systems that combine chemical synthesis with enzymatic catalysis within a cell-free hybrid reactor (Adams et al., 2019; Fricke et al., 2020; Milne et al., 2020). Methods of larger-scale production are available to meet newfound interest in psilocybin and rapid techniques for the assessment of purity on modern instrumentation are desirable. New psilocybin products currently being developed must be evaluated for purity to ensure consistency and quality of research. As the scale of psilocybin production expands through the drug development process, the need for an evaluation of standard analytical methods for everyday analytical platforms is essential.

Analysis of psilocybin has been somewhat overlooked through history because of the controlled nature of the substance. We adapted literature methods (Table S1) for our equipment and found most failed to reproducibly produce a single well-resolved peak for the compound. Many of these methods employed typical $C_{18}$ or $C_{8}$ columns, readily available eluents, and typical temperature programming since these are important criteria for robust HPLC analysis. We found the main source of inaccuracies with certain literature methods were related to either high concentrations of eluent additive employed or unbuffered formic acid use. Psilocybin and psilocin remain infrequently analyzed and standard analytical methods are not well developed or validated. Further, research is partially limited to academic laboratories with special clearance and advanced research equipment. The handful of analytical methods developed in academic settings tend to be more selective and potentially more accurate; however, they are also costly (Tsujikawa et al., 2003; Anastos et al., 2006) and inherently less available to the average analyst. Other quantitative HPLC methods provided sound characterization at the expense of acquisition time. (Tsujikawa et al., 2003; Saito et al., 2005; Anastos et al., 2006; Pellegrini et al., 2013; Pichini et al., 2014; Zhuk et al., 2015; Demmler et al., 2020)

The objective of this research was to achieve a reliable peak shape and maximize performance for the quantitation of psilocybin and psilocin. We aimed to keep the method short for use as a high-throughput purity check. Here, we present our results and discuss HPLC method development along with the pitfalls of commonly used HPLC eluent additives for this analysis. 


\section{Experimental}

\section{Equipment}

An Agilent 1220 Infinity II HPLC equipped with a variable wavelength detector (VWD) set to monitor $220 \mathrm{~nm}$ absorption was used for separation and characterization. HPLC hardware (including pumps and heaters) used were stock equipment only. The column used was a common octadecyl silica column (Agilent Poroshell 120 EC-C18 2.7 $\mu \mathrm{m}$ $3.0 \times 50 \mathrm{~mm}$ ) which was held at $50.0 \pm 0.8^{\circ} \mathrm{C}$ for the duration of the experiments. Manual injection volume was fixed at $5 \mu \mathrm{L}$ by injection loop. The flow rate was set to 1.00 $\mathrm{mL} / \mathrm{min}$ with a set maximum pressure of 400 bar. See Table 1 for the gradient elution program used.

\section{Chemicals and Reagents}

HPLC-grade deionized water and acetonitrile were purchased from Thermo Fisher Scientific and used as eluents without further purification. The aqueous eluent was prepared, as needed, with a concentration of $0.143 \mathrm{mM}$ ammonium formate (Sigma Aldrich, reagent grade, $97 \%$,). Analytical grade formic acid was used for eluents in initial eluent composition experiments (Fisher 99.0+\%, Optima ${ }^{\mathrm{TM}} \mathrm{LC} / \mathrm{MS}$ Grade). Psilocybin (99.977\%) was purchased from Psygen Labs and used without further purification. Blanks analyzed in-between replicates ensured the column was free from impurities for backto-back injections.

\section{Data Processing}

Data were collected using Agilent ChemStation version C.01.10 and processed with a combination of commercial and open-source software. VWD signals for each experiment were extracted using Python 3.9.2 for centre-gravity retention time measurement and peak Gaussian-fit analysis using OriginPro 2021b. Additional figures were produced using Microsoft Excel and Power BI Desktop.

\section{Method Development and Validation}

Psilocybin external standard preparation 
The solid psilocybin (Psygen Labs, 99.977\%) was stored in an airtight container without exposure to light at ambient temperature and otherwise used as received. A stock psilocybin solution was prepared by measuring and dissolving $0.0512 \mathrm{~g}$ of solid psilocybin weighed via analytical mass balance into $100.00 \mathrm{~mL}$ of deionized water measured with a class A volumetric flask. The stock solution was sonicated for 5 minutes to ensure dissolution. Standards used for the calibration curve were prepared gravimetrically at room temperature $\left(22.0^{\circ} \mathrm{C}\right)$ in $\mathrm{HPLC}$ grade water from the $512.0 \mathrm{ppm}$ psilocybin stock solution.

\section{CRM sample preparation}

Quality control samples were prepared by diluting appropriate amounts of psilocybin (Supelco Cerilliant 99.99\%, $1.000 \mathrm{mg} / \mathrm{mL}$ in 50:50 H $\mathrm{H}_{2} \mathrm{O}: \mathrm{MeCN}$ ) and Psilocin (Supelco Cerilliant $99.4 \%, 1.000 \mathrm{mg} / \mathrm{mL}$ in $\mathrm{MeCN}$ ) certified reference materials (CRM). These samples were used to validate the analytical method for performance. Precision was measured through triplicate injections of individual CRM samples (50.0 ppm) which were quantified against pure psilocybin (Psygen Labs, 99.977\%) calibrant solutions prepared in HPLC grade water.

\section{HPLC Parameters}

For interlaboratory comparison and cost-effectiveness, methods involving expensive or time-consuming steps, such as high-resolution mass spectrometric detection or timeconsuming chemical derivatization strategies, were not considered. For similar reasons, and because many laboratories use $\mathrm{C}_{18}$ octadecyl silica HPLC columns, more expensive column chemistries including ion-exchange resins were not considered. As an alternative, we used a conventional (Poroshell 120 EC-C18 2.7 $\mu \mathrm{m} 3.0 \times 50 \mathrm{~mm}$ ) $2.7 \mu \mathrm{m}$ particle $\mathrm{C}_{18}$ column which is best for (U)HPLC performance at lower operational pressures (Agilent Poroshell 120 EC-C18 Threaded Column Data Sheet, 2009). This particular particle size and column chemistry are known to significantly improve reversed-phase chromatography peak resolution and feature high separation efficiency. (Lurie and $\mathrm{Li}$, 2009) $\mathrm{C}_{18}$ columns are preferable to $\mathrm{C}_{8}$ (arguably the next most common HPLC column material) columns for polar organic compound separation. This column chemistry and effects on retention are like that of traditional, fully porous, ZORBAX Eclipse-type $C_{18}$ columns. The choice of column ensured this method is applicable in many laboratories. 
A $1.0 \mathrm{~mL} / \mathrm{min}$ flow rate was used, and the column compartment was set to maintain $50.0^{\circ} \mathrm{C}$ throughout the experiment. Little difference in chromatographic quality was observed at lower flow rates $(0.800 \mathrm{~mL} / \mathrm{min})$, though the high flow rate facilitates an increased sampling rate. The variable wavelength detector (VWD) used is a high sensitivity detector that sacrifices observation of the entire UV-Vis spectrum of an analyte for sensitivity and is intentionally set to monitor a characteristic wavelength for both target analytes.

\section{Gradient program and chromatographic efficiency}

Polar compounds with phosphate functionality (and other moieties with multiple oxygen groups) are difficult to cleanly separate with common $C_{18}$ columns. Highly polar analytes, especially phosphorous-functionalized organics like psilocybin are not wellretained with less polar $\mathrm{C}_{18}$ columns and zwitterionic species are highly water-soluble. As a result, psilocybin elutes at a short retention time ( 0.615 minutes) with high aqueous content mobile phases. Conversely, psilocin is more efficiently eluted using organic mobile phases and clear separation between psilocybin and psilocin may be obtained using an early ( 0.33 mins) switch in eluent polarity (see Table 1 for gradient program). We initially employed HPLC grade methanol but switched to HPLC acetonitrile because of known issues with psilocybin peak shape (Beug and Bigwood, 1981). Additionally, acetonitrile offers reduced solvent background absorption for low-wavelength quantitation, enhanced elution capacity, and reduced operating pressures compared to methanol (Shimadzu, 2007). The organic phase ramp was programmed to switch to the organic phase near the retention time at which psilocybin elutes. By using this approach, psilocybin and psilocin elute efficiently.

Table 1. HPLC gradient elution program

\begin{tabular}{|l|l|l|l|}
\hline $\begin{array}{l}\text { Time } \\
\text { (mins) }\end{array}$ & $\begin{array}{l}\text { \% Eluent A (Deionised } \mathbf{H}_{\mathbf{2}} \mathbf{O}, \\
\mathbf{0 . 1 4 3} \mathbf{~ m M} \text { ammonium } \\
\text { formate) }\end{array}$ & $\begin{array}{l}\text { \% Eluent B } \\
\text { (MeCN) }\end{array}$ & $\begin{array}{l}\text { Flow Rate } \\
(\mathbf{m L} / \mathbf{m i n})\end{array}$ \\
\hline $\mathbf{0}$ & 97 & 3 & 1.00 \\
\hline $\mathbf{0 . 3 3}$ & 97 & 3 & 1.00 \\
\hline $\mathbf{1 . 0 0}$ & 5 & 95 & 1.00 \\
\hline $\mathbf{2 . 0 0}$ & 5 & 95 & 1.00 \\
\hline $\mathbf{3 . 0 0}$ & 95 & 5 & 1.00 \\
\hline
\end{tabular}




\section{Calibration and CRM quantification}

The calibration curve and chromatograms for psilocybin standards are seen in Figures 1 and 2. The run time is short and may feasibly be kept under 1 minute for psilocybin or 2 minutes for psilocybin and psilocin separation. Standards below the lowest concentration reported in the calibration were eliminated as outliers below our method limit of detection (2.10 ppm). The linear fit for this calibration demonstrated good agreement $\left(0.985\right.$ Pearson $\left.R^{2}\right)$ for the concentration range used (2.53-81.9 ppm).

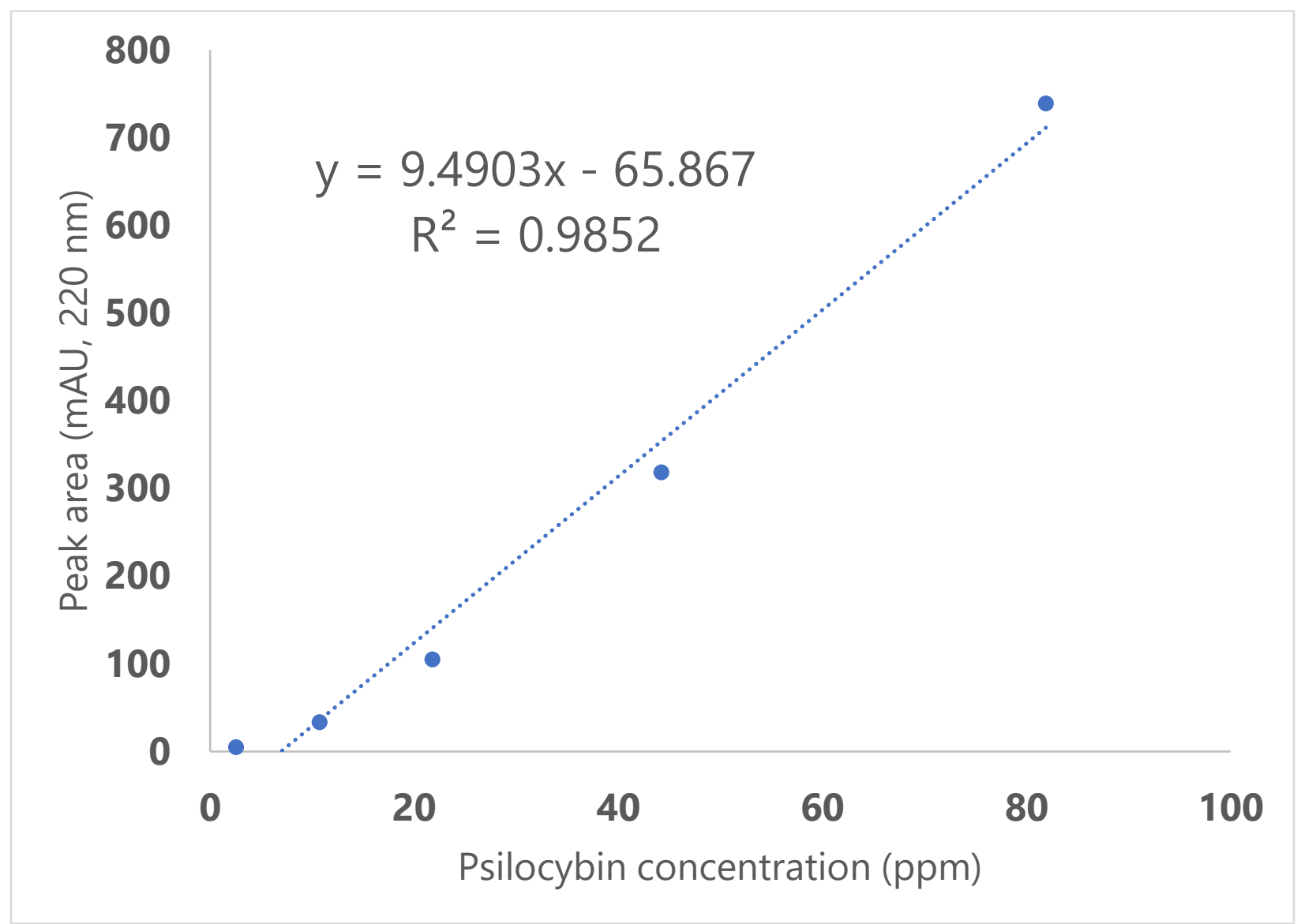

Figure 1. Method calibration curve for Psilocybin measured by HPLC-VWD at $220 \mathrm{~nm}$. 


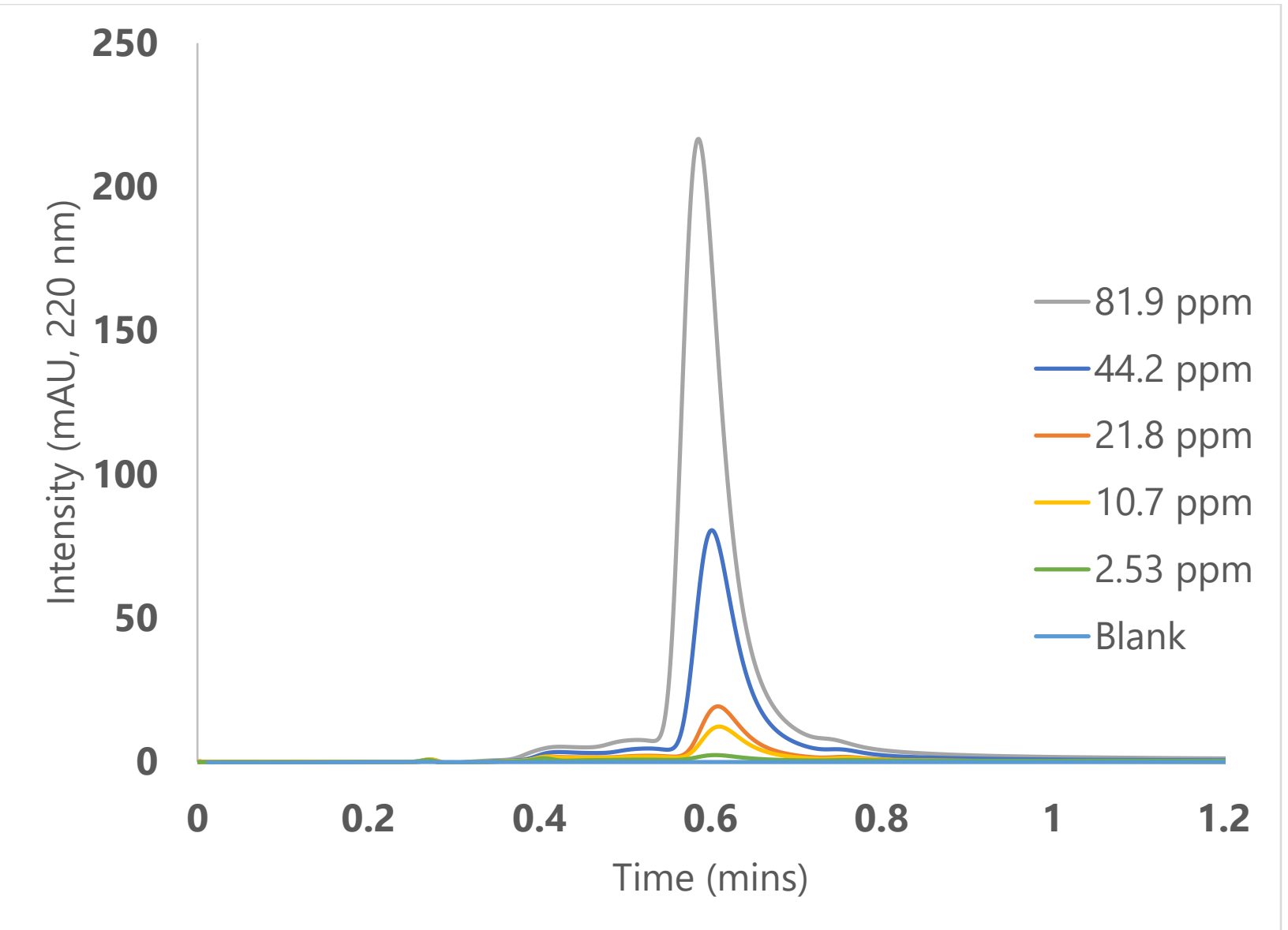

Figure 2. Psilocybin calibrant HPLC chromatogram traces (2.53 - 81.9 ppm Psilocybin)

Quantification of psilocybin and psilocin CRM samples (50.0 ppm) was facilitated using the external calibration standards prepared from solid Psilocybin. Individually prepared samples were made in triplicate and measured with a precision of $0.32 \%$ RSD and $3.5 \%$ bias from expected concentration.

\section{Results and Discussion}

Mobile phases in HPLC are often prepared as aqueous solutions with a variety of additives to prepare a buffer of a suitable $\mathrm{pH}$ range. Psilocybin is an organic zwitterionic compound and rests preferentially in one state over the other depending on the $\mathrm{pH}$ of the eluent (or diluent). In neutral pH deionized water, for example, psilocybin presents itself as a bimodal absorption representing a ratio of charge states present in solution. The difference in coulombic interactions with the stationary phase results in an unacceptable analyte peak shape for reliable quantitation. One charge state is usually fixed (for ionic compounds) by way of eluent additives. Psilocybin has a predicted pKa of 
1.3, 6.5, and 10.4, and both retention time and peak resolution are dramatically affected near these $\mathrm{pH}$ values (Psilocybine - PubChem; Dolan, 2012). For ionizable or permanently charged complexes like psilocybin, eluent $\mathrm{pH}$ must be judiciously prepared for ideal peak shape and resolution. Further, the various methods of preparation or practices may result in some confusion and must therefore be reported clearly to improve reproducibility between labs.

Formic acid is a popular eluent additive used to adjust eluent $\mathrm{pH}$ or protonate organic compounds for mass spectrometric detection. The buffering range of formic acid is between $\mathrm{pH}$ 2.77-4.77 and serves as an appropriate buffer for mild acidification over harsher options like phosphoric acid. Perhaps the most common concentration of formic acid-based eluent is $0.1 \%$ by volume and is sold commercially; although, methods have been reported with using elevated concentrations up to 0.3\% (v/v) (Pichini et al., 2014; Zhuk et al., 2015). However, in our experiments, a standard amount of dilute (0.1\%) formic acid failed to fix the charge state of the analyte and resulted in characteristic twin peaks or poor separation. As well, more dilute formulations suffered from the same lack of resolution. For psilocybin, low buffering agent concentration avoids potential degradation of the analyte as is observed at more extreme $\mathrm{pH}$ levels using formic acid.

Instead, we opted to use ammonium formate (buffer $\mathrm{pH}$ ranges of 2.77-4.77 and 8.2510.25) to reduce the issues observed with pure formic acid. As an eluent additive, suggested concentrations range broadly between 5-300 mM (United Nations, 1989; Saito et al., 2005); however, eluent additives above approximately $0.1 \mathrm{M}$ are susceptible to precipitation and should be used cautiously. For this reason, we successively diluted recommended concentrations of ammonium formate eluent and attempted separation at the reduced concentrations. We found only a modest amount $(0.143 \mathrm{mM}$ reported) of ammonium formate was required for excellent peak shape and performance. The capacity factor for psilocybin with this method was measured as 1.23 , on average. Peak shape for both analytes (psilocybin and psilocin) was separately measured by the average signal of triplicate QC sample injections. The Gaussian fit of this data agrees well $\left(0.967\right.$ Pearson $\left.\mathrm{R}^{2}\right)$ indicating psilocybin resides preferentially in the protonated state. The average QC retention time was measured at 0.615 minutes for psilocybin with a peak width of 0.0504 mins (FWHM). Peak shape and retention time for psilocin were also determined through the average of triplicate psilocin QC sample injections. The Gaussian fit of this peak also agrees well $\left(0.939\right.$ Pearson $\left.R^{2}\right)$ and resolves suitably $(0.125$ 
mins FWHM). No additional peaks were observed for the psilocybin standards or QC samples, or the psilocin QC samples. It is worth noting that psilocybin may be monitored at 268 or $290 \mathrm{~nm}$ for enhanced selectivity (Psilocybin Product Information, 2021) at the cost of sensitivity. For purified samples and this eluent system $\left(\mathrm{H}_{2} \mathrm{O} /\right.$ $\mathrm{MeCN}), 220 \mathrm{~nm}$ is effective for determining psilocybin and psilocin concentration (Tsujikawa et al., 2003). Both compounds absorb strongly at this wavelength and excellent accuracy may be expected at concentrations above a low (2.1 ppm) method limit of detection. The low concentration of ammonium formate used in this method minimizes spectral background, reduces reagent cost for high-throughput laboratories, and is also appropriate for gentle treatment of newer silica columns. For most analytical separations, the concentration and injection volumes used are low (ppm to ppb concentrations and $\mu \mathrm{L}$ scale volumes) and therefore it is possible to use dilute formulations and still provide adequate buffering capacity (Dolan, 2012).

\section{Conclusions}

We developed a robust and fast analytical method for the separation and quantification of psilocybin and psilocin in under 2 minutes using inexpensive equipment, consumables, and reversed phase $\mathrm{C}_{18} \mathrm{HPLC}$. The importance of consistent eluent preparation and issues with standard compositions of formic acid were highlighted in our experiments and prudent eluent preparation. We assume the low pKa of formic acid may cause some amount of degradation via hydrolysis or dephosphorylation. Moreover, unbuffered formic acid may not fix the eluent and analyte $\mathrm{pH}$ and therefore multiple charge states are detected in-flow. Further investigation is currently underway to determine the nature of formic acid's interference with psilocybin. The simple conditions employed here use only a small concentration of ammonium formate as an eluent additive and pure acetonitrile to achieve good quantitative accuracy and precision (0.32\% RSD and $3.5 \%$ bias). High purity solvents and the optimized instrument conditions achieved consistent results with excellent peak shape and a method limit of detection of $2.1 \mathrm{ppm}$.

\section{Acknowledgements}

The authors would like to thank Professor Tao Huan, Dr. Tom Dupree, and Ali Wasti for helpful discussions. 


\section{References}

Adams, A. M., Kaplan, N. A., Wei, Z., Brinton, J. D., Monnier, C. S., Enacopol, A. L., et al. (2019). In vivo production of psilocybin in E. coli. Metab. Eng. 56, 111-119. Agilent Poroshell 120 EC-C18 Threaded Column Data Sheet (2009).

Anastos, N., Lewis, S. W., Barnett, N. W., and Sims, D. (2006). The determination of psilocin and psilocybin in hallucinogenic mushrooms by HPLC utilizing a dual reagent acidic potassium permanganate and tris(2,2'-bipyridyl)ruthenium(II) chemiluminescence detection system. J. Forensic Sci. 51, 45-51. doi:10.1111/j.1556-4029.2005.00033.x. Andén, N. E., Corrodi, H., and Fuxe, K. (1971). Hallucinogenic drugs of the indolealkylamine type and central monoamine neurons. J. Pharmacol. Exp. Ther. 179, 236-249.

Beug, M. W., and Bigwood, J. (1981). Quantitative analysis of psilocybin and psilocin and psilocybe baecystis (singer and smith) by high-performance liquid chromatography and by thin-layer chromatography. J. Chromatogr. A 207, 379-385. doi:10.1016/S00219673(00)88741-5.

Carhart-Harris, R., Giribaldi, B., Watts, R., Baker-Jones, M., Murphy-Beiner, A., Murphy, R., et al. (2021). Trial of Psilocybin versus Escitalopram for Depression. N. Engl. J. Med. 384, 1402-1411. doi:10.1056/nejmoa2032994.

Carhart-Harris, R. L., and Goodwin, G. M. (2017). The Therapeutic Potential of Psychedelic Drugs: Past, Present, and Future. Neuropsychopharmacology 42, 2105-2113. doi:10.1038/npp.2017.84.

Cormier, Z. (2020). Psilocybin Treatment for Mental Health Gets Legal Framework Scientific American. Sci. Am. Available at: https://www.scientificamerican.com/article/psilocybin-treatment-for-mental-healthgets-legal-framework/ [Accessed June 26, 2021].

Daniel, J., and Haberman, M. (2017). Clinical potential of psilocybin as a treatment for mental health conditions. Ment. Heal. Clin. 7, 24-28. doi:10.9740/mhc.2017.01.024. Demmler, R., Fricke, J., Dörner, S., Gressler, M., and Hoffmeister, D. (2020). S-Adenosyl-IMethionine Salvage Impacts Psilocybin Formation in "Magic" Mushrooms.

ChemBioChem 21, 1364-1371. doi:10.1002/cbic.201900649.

Dolan, J. (2012). A Guide to HPLC and LC-MS Buffer Selection. Www.Ace-Hplc.Com. Available at: www.ace-hplc.com [Accessed June 27, 2021].

Fricke, J., Kargbo, R., Regestein, L., Lenz, C., Peschel, G., Rosenbaum, M., et al. (2020). Scalable hybrid synthetic/biocatalytic route to psilocybin. Chem. Eur. J. 
Fricke, J., Lenz, C., Wick, J., Blei, F., and Hoffmeister, D. (2019). Production Options for Psilocybin: Making of the Magic. Chem. - A Eur. J. 25, 897-903.

doi:10.1002/chem.201802758.

Geiger, H. A., Wurst, M. G., and Daniels, R. N. (2018). DARK Classics in Chemical Neuroscience: Psilocybin. ACS Chem. Neurosci. 9, 2438-2447.

doi:10.1021/acschemneuro.8b00186.

Gouzoulis-Mayfrank, E., Schreckenberger, M., Sabri, O., Arning, C., Thelen, B., Spitzer, M., et al. (1999). Neurometabolic effects of psilocybin, 3, 4-

methylenedioxyethylamphetamine (MDE) and d-methamphetamine in healthy volunteers: a double-blind, placebo-controlled PET study with [18F] FDG.

Neuropsychopharmacology 20, 565-581.

Lurie, I., and Li, L. (2009). Use of high-temperature liquid chromatography with sub-2m particle C18 columns for the analysis of seized drugs. J. Liq. Chromatogr. Relat. Technol. 32, 2615-2626. doi:10.1080/10826070903245516.

Milne, N., Thomsen, P., Knudsen, N. M., Rubaszka, P., Kristensen, M., and Borodina, I. (2020). Metabolic engineering of Saccharomyces cerevisiae for the de novo production of psilocybin and related tryptamine derivatives. Metab. Eng. 60, 25-36.

Molecule of the Week Archive - Psilocybin (2017). Am. Chem. Soc.

doi:10.1021/ac60138a766.

Passie, T., Seifert, J., Schneider, U., and Emrich, H. M. (2002). The pharmacology of psilocybin. Addict. Biol. 7, 357-364. doi:10.1080/1355621021000005937.

Pellegrini, M., Rotolo, M. C., Marchei, E., Pacifici, R., Saggio, F., and Pichini, S. (2013).

Magic truffles or Philosopher's stones: A legal way to sell psilocybin? Drug Test. Anal. 5, 182-185. doi:10.1002/dta.1400.

Pichini, S., Marchei, E., García-Algar, O., Gomez, A., Di Giovannandrea, R., and Pacifici, R. (2014). Ultra-high-pressure liquid chromatography tandem mass spectrometry determination of hallucinogenic drugs in hair of psychedelic plants and mushrooms consumers. J. Pharm. Biomed. Anal. 100, 284-289. doi:10.1016/j.jpba.2014.08.006.

Psilocybin Product Information (2021). Cayman Chem. Available at:

https://www.caymanchem.com/pdfs/15695.pdf.

Psilocybine - PubChem PubChem. Available at:

https://pubchem.ncbi.nlm.nih.gov/compound/psilocybine [Accessed June 27, 2021].

Saito, K., Toyo'oka, T., Kato, M., Fukushima, T., Shirota, O., and Goda, Y. (2005).

Determination of psilocybin in hallucinogenic mushrooms by reversed-phase liquid 
chromatography with fluorescence detection. Talanta 66, 562-568.

doi:10.1016/j.talanta.2004.11.031.

Shimadzu (2007). Tips for practical HPLC analysis-Separation Know-how-Shimadzu LC World Talk Special Issue Volume 2. 2.

Shirota, O., Hakamata, W., and Goda, Y. (2003). Concise large-scale synthesis of psilocin and psilocybin, principal hallucinogenic constituents of 'magic mushroom'. J. Nat. Prod. 66, 885-887. doi:10.1021/np030059u.

Tsujikawa, K., Kanamori, T., Iwata, Y., Ohmae, Y., Sugita, R., Inoue, H., et al. (2003). Morphological and chemical analysis of magic mushrooms in Japan. Forensic Sci. Int. 138, 85-90. doi:10.1016/j.forsciint.2003.08.009. United Nations (1989). Recommended methods for testing Peyote Cactus (Mescal Buttons)/Mescaline and Psilocybe Mushrooms/Psilocybin. 1-42. Vollenweider, F. X. (1998). Advances and pathophysiological models of hallucinogenic drug actions in humans: a preamble to schizophrenia research. Pharmacopsychiatry. Zhuk, O., Jasicka-Misiak, I., Poliwoda, A., Kazakova, A., Godovan, V. V., Halama, M., et al. (2015). Research on acute toxicity and the behavioral effects of methanolic extract from psilocybin mushrooms and psilocin in mice. Toxins (Basel). 7, 1018-1029. doi:10.3390/toxins7041018. 